\title{
SEPARATE FINGER REPRESENTATIONS AT THE HUMAN SECOND SOMATOSENSORY CORTEX
}

\author{
R. HARI, ${ }^{* \dagger}$ H. HÄMÄLÄINEN, + M. HÄMÄLÄINEN, ${ }^{+}$J. KEKONI, + \\ M. SAMS* and J. TIIHONEN* \\ *Low Temperature Laboratory, Helsinki University of Technology, SF-02150 Espoo, Finland \\ $\ddagger$ Department of Psychology, University of Helsinki, 00170 Helsinki, Finland
}

\begin{abstract}
We recorded neuromagnetic responses of the second somatosensory cortex in healthy humans. Cutaneous electrical stimulation of fingers elicited a response around $100 \mathrm{~ms}$. with a field pattern agreeing with activation of the second somatosensory cortex in the upper bank of the Sylvian fissure. In an oddball paradigm, with standards presented to the thumb and deviants $(10 \%)$ to the middle finger, or vice versa, the second somatosensory cortex responses to deviants were almost three times as high in amplitude as those to standards. A similar amplitude enhancement was obtained when the deviants were presented in the absence of the intervening standards but with the same interstimulus interval.

The results indicate that an accurate functional representation of different body areas is maintained at the human second somatosensory cortex.
\end{abstract}

The first (primary) somatosensory cortex (SI) contains multiple representations of the body surface ${ }^{12.17}$ and is involved in accurate analysis of different features of tactile stimuli. In contrast, the functions of the second somatosensory cortex (SII) are poorly understood' and even the organization of the afferent pathways is still controversial. SII probably receives direct afferentation from thalamus and relayed input through SI. 2.20

The existence of the human SII, buried within the upper bank of the Sylvian fissure, was first shown in intraoperative cortical recordings by Penfield and Jasper. ${ }^{19}$ The first non-invasive recordings, by means of magnetoencephalography (for revicws of the method, see Refs 7-9, 25) showed activity at SII $110-140 \mathrm{~ms}$ after median nerve stimulation at the wrist or peroneal nerve stimulation at the ankle. ${ }^{6.10 .14 .23}$ Activity at the hand SI produces a characteristic "dipolar" pattern of the magnetic field component perpendicular to the skull, with two extrema of opposite polarities, one at the upper and the other at the lower end of the Rolandic fissure. ${ }^{7.24 .26}$ The field distribution of SII-responses was clearly different, with two extrema of opposite polarities at the ends of the Sylvian fissure. Ipsi- and contralateral stimuli, presented either to the upper or lower limbs, produced similar responses, further supporting activation of SII.

In the present work we studied magnetic responses $f$ SIl to cutaneous stimulation of fingers. Special

†To whom correspondence should be addressed.

Abbreviations: DC-SQUID, direct current superconducting quantum interference device; EEG, electroencephalography: EOG. electro-oculogram; MEG, magnetoencephalography: SI, primary somatosensory cortex: SII, second somatosensory cortex. emphasis was put on their sensitivity to infrequent changes in the stimulus location from one finger to another.

\section{EXPERIMENTAL PROCEDURES}

Five healthy adults were studied in the Otaniemi magnetically shielded room. ${ }^{15}$ Three of them were authors and two were paid students. The magnetic field outside the head was measured with a seven-channel first-order direct current superconducting quantum interference device (DC-SQUID) gradiometer $^{16}$ (field sensitivity $5-6 \mathrm{fT} / \sqrt{\mathrm{Hz}}$ ). The pick-up coils of the device are placed on a hexagonal grid $36.5 \mathrm{~mm}$ from each other and cover an area with a diameter of $93 \mathrm{~mm}$. During the measurements the subject was lying with the head supported by a vacuum cast. The locations and orientations of the sensors with respect to external landmarks of the skull were measured by recording magnetic signals resulting from the activation of a set of small coils placed on known locations on the scalp. Some control experiments were carried out with a new 24-channel SQUID-device, ${ }^{13}$ which will be described in Results.

The stimuli were $0.3 \mathrm{~ms}$ constant-current pulses delivered on the volar skin of the distal thumb and the middle finger (Grass S88 stimulator, Grass SIU 4678 isolation unit, and Grass CCU (A constant current unit). The pads ( $\phi=6 \mathrm{~mm})$ of the stimulus electrode were separated by $25 \mathrm{~mm}$. In most experiments, an oddball paradigm was used with standard stimuli [probability $(P)=0.9$ ] presented to the thumb, and the deviants $(P=0.1)$ to the middle finger, or vice versa; each deviant was randomly followed by three to 15 standards with an even distribution. The interstimulus interval was $555 \mathrm{~ms}$. The subject was instructed either to count the deviants (attend condition) or to ignore all stimuli (ignore condition). In addition. several control experiments were conducted.

The recording passband was $0.05-500 \mathrm{~Hz}(3 \mathrm{~dB}$ points, high-pass roll-off $35 \mathrm{~dB} /$ decade and low-pass over $80 \mathrm{~dB}$ decade). The signals were digitized at $2000 \mathrm{~Hz}$ and averaged on-line; the analysis period of $256 \mathrm{~ms}$ included a prestimulus baseline of $40 \mathrm{~ms}$. Responses with amplitudes exceeding $150 \mu \mathrm{V}$ in the simultaneously recorded vertical electrooculogram (EOG) were rejected from the analysis, as well as the first two responses of each stimulus block. Further, each response to the standard immediately after the deviant was 


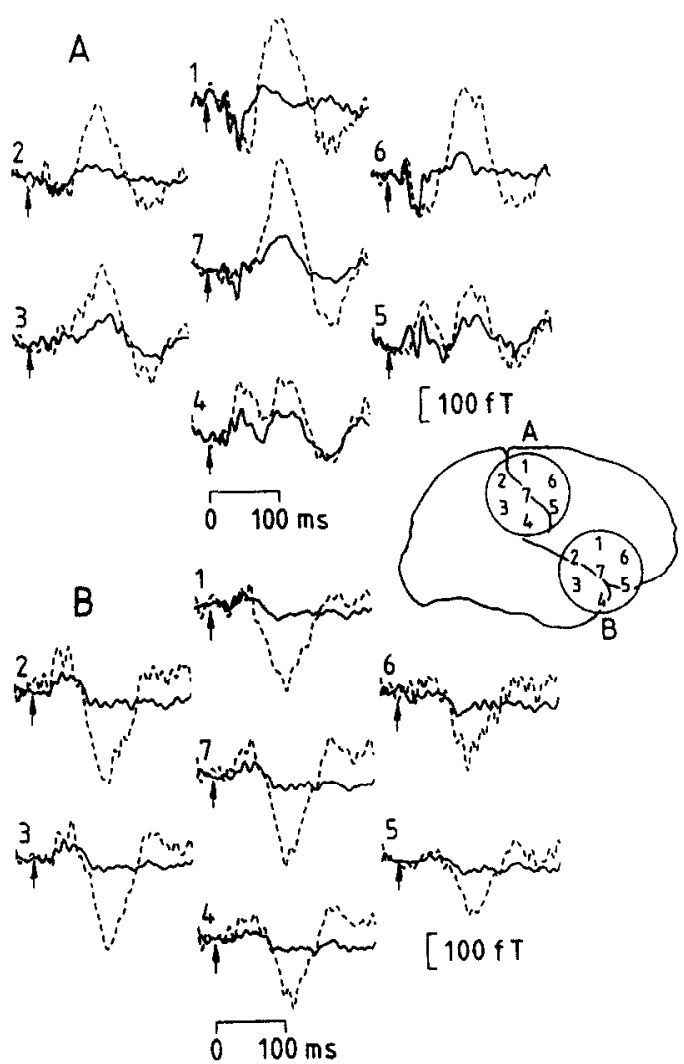

Fig. 1. Seven-channel recordings at two locations (shown schematically on the inset brain) to electric puises presented on left thumb (standards, continuous lines) and middle finger (deviants, dashed lines). The responses have been digitally filtered with a passband of $0.05-90 \mathrm{~Hz}$. The arrows show the time of stimulus presentation.

omitted from the analysis. The peak-to-peak amplitudes of the averaged EOG did not exceed $10 \mu \mathrm{V}$. About 360 responses for standards and $\mathbf{4 0}$ for deviants were averaged at each location. Response amplitudes were measured with respect to the 40 -ms prestimulus baseline.

To determine the sources, responses were recorded from 35 to 49 locations over the right hemisphere. Isocontour maps were constructed by projecting the measurement locations to a plane. If the pattern was dipolar the equivalent dipole was determined with a least-squares search, using the original data and the measured locations and orientations of the sensors. A spherical volume conductor model was used with a radius corresponding to the local radius of curvature of the measurement area. In this procedure the effects of source and volume currents on the measured field are properly taken into account."

\section{RESULTS}

The responses of one subject when the standards were presented to the left thumb and the deviants to the middle finger are shown in Fig. 1. Some early responses, like that peaking at $30 \mathrm{~ms}$, have different polarities at different channels at the posterior measurement area, thereby suggesting that the recording has been made directly over the hand SI area. These early deflections have been described in detail earlier. ${ }^{24}$ The response to standards contains main peaks at 45 and $105 \mathrm{~ms}$; the relative amplitudes of these responses vary at different measurement locations. The 100-ms response is clearly enhanced for the deviant stimuli. The mean amplitude was almost three times as high to the deviants as to the standards (five subjects; $P<0.005$, two-tailed $t$-test for pair differences).

The field patterns of both deflections were dipolar but clearly different (Fig. 2). During the 45-ms response the pattern can be explained by activation of the SI hand area in the posterior wall of the Rolandic fissure. During the latter response the orientation of the equivalent current source is different and the location is more anterior and lateral, agreeing with the site of SII. The source locations were different for the early and late responses in all subjects; the separation varied between 15 and $45 \mathrm{~mm}$. However, the sources did not differ signifcantly between responses to standards and deviants. The field was strongest during the 105-ms deflection to deviants in the attend condition.

In Fig. 1 the early response, also, was larger to deviants than to standards, but in the group means, shown in Table 1, this difference was not statistically significant and there were no significant latency differences between the conditions. The amplitude difference between responses to standards and deviants was significantly stronger for the late than for the early deflection (the mean increases $166 \%$ and $45 \%$, respectively, when the attend and ignore data were pooled; $P<0.001$, two-tailed $t$-test for pair differences). Similar amplitude enhancement of the SII-responses was obtained when the standard and deviant stimuli were reversed. Additional recordings in one subject showed increased SII-responses to deviants even when the standards were presented to the proximal part of the middle finger and the deviants to the distal one; the subject had difficulties in discriminating the stimuli.

The control recordings with the 24-channel device, illustrated in Fig. 3, show that responses to deviants presented alone, without the intervening standards, are very similar to responses to deviants among the standards. The late deflections were evoked by ipsilateral stimulation, as well, although with clearly lower amplitude and a 5-10 ms longer latency. For the data of Fig. 3 the strength of the equivalent dipole was $33 \mathrm{nA} \cdot \mathrm{m}$ for contralateral and $20 \mathrm{nA} \cdot \mathrm{m}$ for ipsilateral deviants among standards. These values were three times as large as those for the standard responses whereas no such increase was observed during the $45-\mathrm{ms}$ response. Similarly as the $20-\mathrm{ms}$ deflection ( $\mathrm{N} 20 \mathrm{~m}$ ), evoked by mixed median nerve stimulation at the wrist, ${ }^{24}$ the $45-\mathrm{ms}$ response was evoked by contralateral stimuli, only.

The amplitude difference between the responses to standards and deviants was seen both during the ignore and the attend conditions. Attention increased some amplitudes slightly (Table 1), most clearly those of the $100-\mathrm{ms}$ responses to the deviants, but the mean differences did not reach statistical significance. 


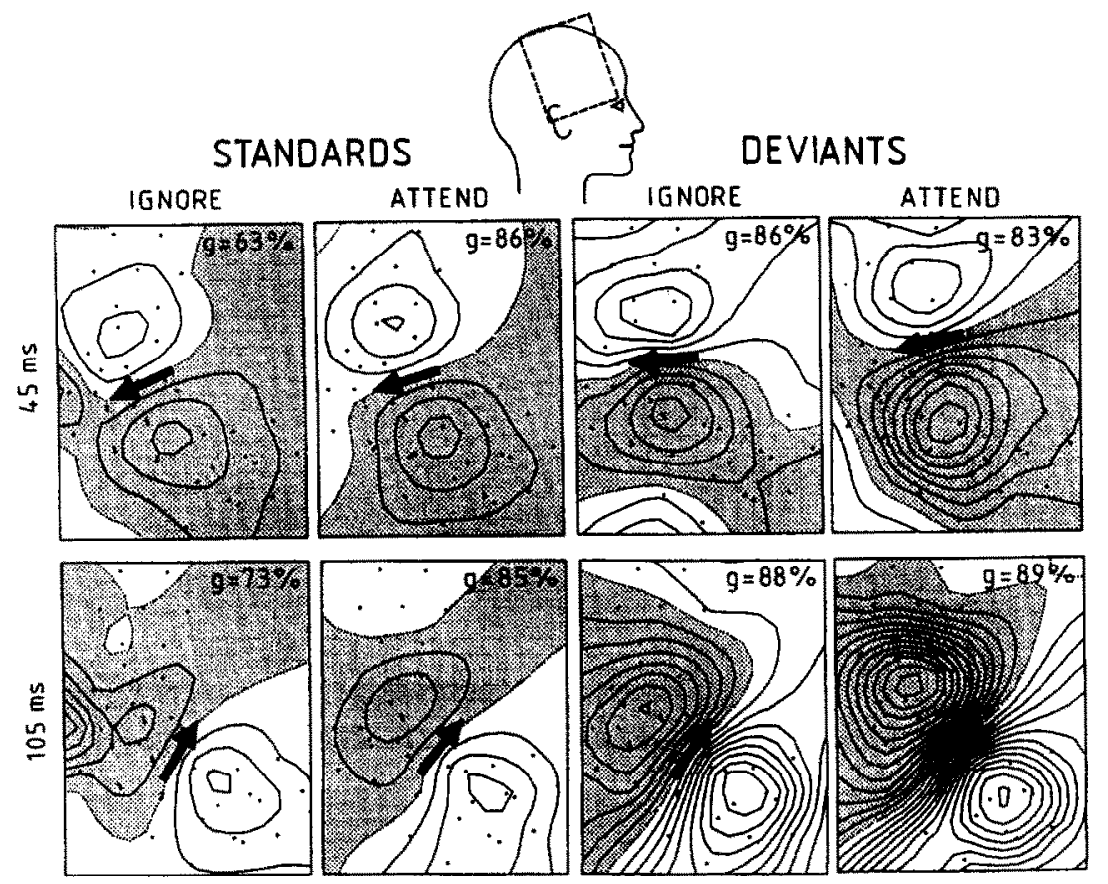

Fig. 2. Field patterns of one subject during the 45- and 105-ms deflections for standards (thumb) and deviants (middle finger) in the ignore and attend conditions. The maps are projections to a plane from the signals measured on the curved surface over the head. The dots show the measurement locations over the right hemisphere (see the schematic head). The isocontour lines are separated by $20 \mathrm{~T}$. The shadowed area indicates fux out of the head and the white area fux into the head. The arrows show the locations and orientations of the equivalent dipoles. The $\boldsymbol{g}$-values refer to the goodness-of-fit of the dipole model. In this subject the sources of the 105-ms deflection were systematically about $2 \mathrm{~cm}$ deeper than those of the 45-ms deflection.

In three subjects, however, the amplitude increase clearly exceeded the noise level, estimated on the basis of the standard errors of the mean of the averaged responses.

\section{DISCUSSION}

The present data agree with earlier results $5^{6.10,14.23}$ confirming that magnetoencephalography (MEG) offers a tool to explore the human SII and to obtain information about its role in the processing of tactile information. Due to its anatomical location, the human SII area has been difficult to study even in intraoperative cortical recordings. For example, Lüders et al. ${ }^{18}$ observed corticographic activity from SII in one out of their 25 patients, only. The SII-activity of that patient occurred already around
$20 \mathrm{~ms}$. Since MEG is mainly sensitive to tangential current sources, 7.9 .25 the absence of such early magnetic responses at SII might be due to radial source orientation.

The responses of SII were strongly, and more clearly than those from SI, enhanced by small changes in the stimulus location, even if the subjective detection of the change was difficult. However, since the responses to deviants were not affected by the standard stimuli, the observed amplitude increase seems not to be specific to stimulus change per se. A simpler explanation is that standards and deviants activate independent neuronal populations, and that the amplitude differences result from differences in stimulus repetition rates. The findings therefore suggest that the representations of nearby body sites, like fingers, are functionally clearly separate at SII. The sharp tuning of body representation is in line

Table 1. The mean ( \pm S.E.M.) latencies (in $\mathrm{ms}$ ) and amplitudes (in $\mathrm{T}$ ) of five subjects for the early and late responses for standards and deviants during the ignore and attend conditions

\begin{tabular}{lcccr}
\hline \multirow{2}{*}{ Condition } & \multicolumn{2}{c}{ Early } & \multicolumn{2}{c}{ Late } \\
\hline lgnore, standard & $44.7 \pm 1.4$ & $68.9 \pm 24.0$ & $113.6 \pm 5.9$ & $86.5 \pm 24.1$ \\
Attend, standard & $45.4 \pm 1.2$ & $83.3 \pm 35.8$ & $112.1 \pm 3.5$ & $77.0 \pm 19.2$ \\
lgnore, deviant & $50.2 \pm 2.1$ & $108.6 \pm 37.0$ & $100.2 \pm 7.5$ & $199.3 \pm 35.2$ \\
Attend, deviant & $46.6 \pm 2.9$ & $111.1 \pm 33.9$ & $103.2 \pm 8.8$ & $234.1 \pm 17.3$ \\
\hline
\end{tabular}

The measurements were made from one representative channel at the temporal area. 


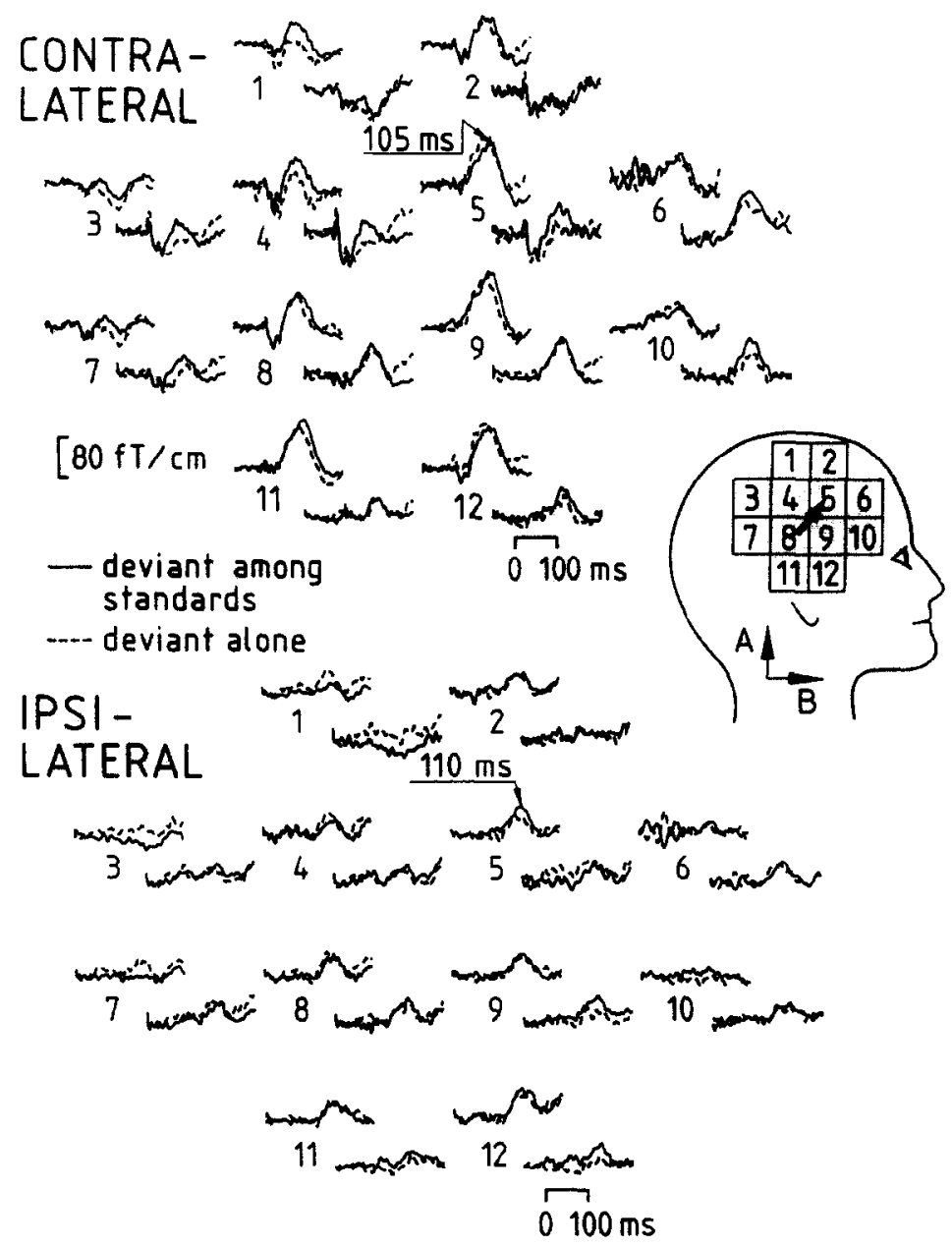

Fig. 3. Responses to the deviants in the presence (continuous lines) and absence (dashed lines) of the intervening standards. The measurement was made with the 24-channel gradiometer recently constructed in our laboratory. "This device uses a novel flux transformer configuration: instead of recording the field

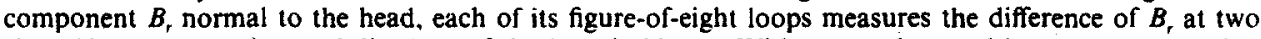
sites, $13 \mathrm{~mm}$ apart; the total diameter of the loop is $30 \mathrm{~mm}$. With two orthogonal loops at one location the two off-diagonal field gradients $\partial B_{r} / \partial x$ and $\partial B_{r} / \partial y$ are obtained at 12 locations (shown in the inset head) simultaneously. While the axial magnetometer detects the field extrema on both sides of the dipole the 24-SQUID planar gradiometer records the largest signal just above the dipolar source. In this figure the maximum total signals $\left(\sqrt{(\partial B, / \partial x)^{2}+(\partial B, / \partial y)^{2}}\right)$ are obtained at pair 9 for the late and at pair 4 for the early deflection; these recording sites are $4.2 \mathrm{~cm}$ from each other. For each pair of traces, the upper ones show the field gradient in vertical direction ( $A$ on the schematic head) and the lower traces in the horizontal direction (B). The arrow in the head shows the approximate location and orientation of the equivalent source for the contralateral $100-\mathrm{ms}$ response. The passband is $0.05-90 \mathrm{~Hz}$, except for pairs 6 which have been low-pass filtered at $45 \mathrm{~Hz}$ due to $50-\mathrm{Hz}$ interference.

with the proposed involvement of SII in tactile learning and memory $;^{4}$ such functions necessitate an accurate trace of the stimulation to be maintained for some time.

SI and SIl are densely interconnected " but their functional relationship is still controversial. SI seems to be mainly involved with accurate analysis of sensory input associated with active touch, i.e. when rapid interaction between the sensory and motor cortices and subsequent feature analysis is necessary, whereas SII combines activity from both sides of the body. Unit activity has been shown to increase at SII when the monkey attends to vibratory stimuli. ${ }^{21}$
A similar trend was seen in the present study but the effect was weak and depended on the subject; one reason for this might be our very easy detection task.

Our results also have implications to electric scalp recordings, since somatosensory evoked potentials, due to their poor spatial resolution, reflect activity of both SI and SII. Several authors have observed the task sensitivity of the 100 -ms somatosensory evoked potential, which has therefore bcen called "cognitive P100" (see e.g. Ref. 3). The present results suggest that the electric P100 is generated at SII (see also Ref. 5), and should therefore be 
considered clearly distinct from the responses originating at SI. Reinterpretation of some earlier electric evoked potential studies within this framework seems necessary.
Acknowledgements-This study was supported by the Academy of Finland and by the Körber Foundation (Hamburg). We thank Mr Seppo Ahlfors for designing some software and Visa Vilkman for expert help with intrumentation.

\section{REFERENCES}

1. Burton H. (1986) Second somatosensory cortex and related areas. In Cerebral Cortex (eds Jones E. G. and Peters A.), pp. 31-98. Plenum Press, New York.

2. Burton H. and Robinson C. J. (1987) Responses in the first and second somatosensory cortical area in cats during transient inactivation of the other ipsilateral area with lidocain hydrochloride. Somatosens. Res. 4, $215-236$.

3. Desmedt J. E., Nguyen T. H. and Bourguet M. (1983) The cognitive P40, N60 and P100 components of somatosensory evoked potentials as the earliest electrical signs of sensory processing in man. Electroenceph. clin. Neurophysiol. 56, 272-282.

4. Garcha H. S. and Ettlinger G. (1978) The effects of unilateral or bilateral removals of the second somatosensory cortex (area SII): a profound tactile disorder in monkeys. Cortex 14, 319-326.

5. Hämäläinen H., Kekoni J., Sams M., Reinikainen K. and Näätänen R. (1990) Human somatosensory evoked potentials to mechanical pulses and vibration: contributions of SI and SII somatosensory cortices to P50 and PI00 components. Eleciroenceph. clin. Neurophysiol. 75, 13-21.

6. Hari R., Hämäläinen M., Kaukoranta E., Reinikainen K. and Teszner D. (1983) Neuromagnetic responses from the second somatosensory cortex in man. Acta neurol. scand. 68, 207-212.

7. Hari R. and IImoniemi R. J. (1986) Cerebral magnetic fields. CRC Crit. Rev. biomed. Engng 14, 93-126.

8. Hari R. and Kaukoranta E. (1985) Neuromagnetic studies of the somatosensory system. Prog. Neurobiol. 24, $233-256$.

9. Hari R. and Lounasmaa O. V. (1989) Recording and interpretation of cerebral magnetic fields. Science 244, $432-436$.

10. Hari R., Reinikainen K., Kaukoranta E., Hämäläinen M., Ilmoniemi R., Penttinen A., Salminen J. and Teszner D. (1984) Somatosensory evoked cerebral magnetic fields from SI and SII in man. Electroenceph. clin. Neurophysiol. 57, 254-263.

11. Jones E. G. and Powell T. P. S. (1968) The ipsilateral cortical connections of the somatic sensory areas in the cat. Brain Res. 9, $71-94$.

12. Kaas J. H., Sur M., Nelson R. J. and Merzenich M. M. (1981) The postcentral somatosensory cortex: multiple representations of the body in primates. In Cortical Sensory Organization, Vol. 1, Multiple Somatic Areas (ed. Woolsey C. N.), pp. 29-45. Humana Press, Clifton, NJ.

13. Kajola M., Ahlfors S.. Enholm G. J., Hällström J., Hämäläinen M. S. and Ilmoniemi R. I. (1990) A 24-channel magnetometer for brain research. In Adtances in Biomagnetism (eds Williamson S. J. et al.). Plenum Press, New York (in press).

14. Kaukoranta E., Hari R.. Hämäläinen M. and Huttunen J. (1986) Cerebral magnetic fields evoked by peroneal nerve stimulation. Somatosens. Res. 3, 309-321.

15. Kelhä V. O., Pukki J. M., Peltonen R. S., Penttinen A. J., Ilmoniemi R. J. and Heino J. J. (1982) Design, construction, and performance of a large volume magnetic shield. IEEE Trans. Magn. 18, 260-270.

16. Knuutila J., Ahlfors S., Ahonen A., Hällström J., Kajola M., Lounasmaa O. V., Tesche C. and Vilkman V. (1987) A large-area low-noise seven-channel DC SQUID magnetometer for brain research. Rev. Sci. Instr. 58, 2145-2156.

17. LaMotte R. H. and Mountcastle W. B. (1979) Disorders in somesthesis following lesions of parietal lobe. $J$. Neurophysiol. 42, 400-419.

18. Lüders H., Lesser R. P., Dinner D. S., Hahn J. F., Salanga V. and Morris H. H. (1985) The second sensory area in human: evoked potential and electrical stimulation studies. Ann. Neurol. 17, 177-184.

19. Penfield W. and Jasper H. (1954) Epilepsy and the Functional Anatomy of the Human Brain. Little, Brown and Company, Boston.

20. Pons T. P., Garraghty P. E., Friedman D. P. and Mishkin M. (1987) Physiological evidence for serial processing in somatosensory cortex. Science $237,417-419$.

21. Poranen A. and Hyvärinen J. (1982) Effects of attention on multiunit responses to vibration in the somatosensory regions of the monkey brain. Electroenceph. clin. Neurophysiol. 53, 525-537.

22. Sarvas J. (1987) Basic mathematical and electromagnetic concepts of the biomagnetic inverse problem. Phys. med. Biol. $32,11-22$.

23. Teszner D., Hari R., Nicolas P. and Varpula T. (1983) Somatosensory evoked magnetic fields: mapping and the influence of stimulus repetition rate. Nuovo Cimento 2D, 429-437.

24. Tiihonen J.. Hari R. and Hämäläinen M. (1989) Early deflections of cerebral magnetic responses to median nerve stimulation. Electroenceph. clin. Neurophysiol. 74, 290-296.

25. Williamson S. J. and Kaufman L. (1981) Biomagnetism. J. Magn. Magn. Mat. 22, 129-202.

26. Wood C. C., Cohen D., Cuffin B. N., Yarita M. and Allison T. (1985) Electric sources in the human somatosensory cortex: identification by combined magnetic and potentials field recordings. Science 227, 1051-1053. 